\title{
Transgene chgH-rfp expression at developmental stages and reproductive status in marine medaka (Oryzias dancena)
}

\author{
Young Sun Cho and Yoon Kwon Nam ${ }^{*}$
}

\begin{abstract}
Background: The transgenic approach using estrogen-responsive regulator in fish has been given much attention as a potential means to detect and/or address estrogen-related aquatic pollutions. In order to address the development stage- and reproduction status-dependent expression patterns of the chgH-rfp transgene (red fluorescent protein transgene driven by choriogenin $\mathrm{H}$ promoter) in marine medaka Oryzias dancena, naturally occurring red fluorescent protein (RFP) signals under non-exposed conditions as well as the transgenically induced RFP signals under estrogen-exposed conditions were assayed.

Results: Female transgenics begun to show naturally occurring RFP signals from the age of 7 weeks post hatching (WPH) without experimental estrogen exposure. Afterward, these RFP signals in female transgenics became robust with the progress of ovarian maturation. On the other hand, male transgenics did not show any naturally occurring RFP signal under non-exposed conditions irrespective of developmental stages and maturation statue. Upon exposures using estradiol-17ß (E2) and 17a-ethinylestradiol (EE2), RFP signals were significantly induced specifically in the livers of transgenic males.
\end{abstract}

Conclusions: Male chgH-rfp transgenics were able to keep the "off" state of RFP expression during their entire life cycle unless exposed to exogenous estrogens. Owing to their tight regulation capability of estrogen-responsive transgene, transgenesis of chgH-rfp in male marine medaka could offer a useful model system for future ecotoxicogenomic studies regarding estrogenicity-related issues in aquatic and marine environments.

Keywords: Estrogen-responsive transgenic, Marine medaka Oryzias dancena, RFP, Choriogenin

\section{Background}

The transgenic approach using estrogen-responsive transgene in a small-sized aquarium fish species has been given much attention as a novel means to detect and/or address estrogen-related aquatic pollutions (Bogers et al. 2006; Chen et al. 2010). Usually, this approach is based on the specific induction of fluorescent phenotype in the fish under the control of an estrogenresponsive regulator. When the transgenic fish are exposed to waterborne estrogenicity, the fluorescent phenotype could be achieved through the induced expression of a transgenically introduced fluorescent protein gene in the target $\operatorname{organ}(\mathrm{s})$ (Chen et al. 2010).

\footnotetext{
* Correspondence: yoonknam@pknu.ac.kr

Department of Marine Bio-Materials and Aquaculture, Pukyong National University, Busan 48513, South Korea

Transgene regulators with this purpose have often been originated from the promoters of genes encoding proteins associated with the ovarian maturation, such as choriogenin (egg envelope protein), vitellogenin (yolk protein), or estrogen receptor (Bogers et al. 2006; Scholz et al. 2005; Stegeman et al. 2010). Potentially advantageous merits of such a transgenic approach over traditional methods using water chemistry or quantitative assay of endogenous gene transcripts include the considerable simplicity and brevity of the analysis procedure, reasonable sensitivity, and/or the possible integration of toxicodynamic and toxicokinetic effects (Scholz et al. 2005; Stegeman et al. 2010).

Several estrogen-responsive transgenic lines have been reported in popularly known model fish species, zebrafish (Danio rerio) and Japanese medaka (Oryzias latipes) (Chen et al. 2010; Zeng et al. 2005; Salam et al. 2008). 
Recently, a truly euryhaline medaka species (Oryzias dancena) has been proposed as a novel and promising platform for estrogen-responsive transgenesis. The extremely high osmoregulatory capability of this species could facilitate the practical application of estrogenresponsive transgenic strains to a wide range of environmental salinity, as evidenced in the established transgenic strains carrying the red fluorescent protein (RFP) gene under the control of endogenous choriogenin $\mathrm{H}$ promoter (chgH-rfp transgenics) (Cho et al. 2013; Nam et al. 2015). Although the potential usefulness of $c h g H$ $r f p$ transgenic marine medaka has been successfully demonstrated (Cho et al. 2013), performance data on their estrogen-responsive function have been limited only to hatchling and larval stages. However, for extended applications of estrogen-responsive transgenics to ecotoxicological or ecotoxicogenomic studies, much more investigations on transgenic functions taking into account the various biological factors such as age, sex, and reproductive status should be needed. For example, due to the intrinsic nature of the transgenic regulator (choriogenin $\mathrm{H}$ promoter) used in marine medaka, female chgH-rfp transgenics are expected to display a naturally occurring fluorescent phenotype even under non-exposed conditions when they reach the stage of ovarian maturation since the synthesis of choriogenins in the liver would be an essential requirement for the formation of egg membrane. In the case of males also, a previous study has shown that marine medaka adult males could express a small amount of $c h g H$ messenger RNAs (mRNAs) in their liver under normal culture conditions, although the fate of the transcribed products in males has not been clearly elucidated yet (Lee et al. 2012). Further, exogenous administration of estrogens has potentially induced de novo synthesis of chgH mRNAs in several male tissues, although the quantities of $c h g H$ transcripts induced in non-liver tissues were significantly smaller than that in the liver (Lee et al. 2012). Collectively, previous findings have led the need for empirical testing to examine whether or not the regulation of the $\operatorname{chgH}$ gene at the transcription level in male tissues would affect the fluorescent phenotype of chgH-rfp transgenic males under both non-exposed and estrogenexposed conditions.

Based on these, the objective of this study was to answer two specific questions in order to investigate the functional evaluation of chgH-rfp transgene in grown-up marine medaka for future applications of this transgenic model strain. The first question is "When do chgH-rfp transgenic marine medaka females begin to show the naturally occurring fluorescence signals during their normal grow-out period?" On the other hand, the second question is "Do transgenic males keep the fluorescencenegative phenotype throughout their life cycle unless they are exposed to exogenous estrogen, and if so, do they show tissue-specific induction of transgenic fluorescent signals in response to estrogen exposure?"

\section{Methods}

\section{Transgenic fish strain}

The transgenic marine medaka strain used in this study had been generated by microinjection of the red fluorescent protein gene ( $r f p$; Takara Bio USA Inc., Mountain View, CA, USA) driven by a 2.8 -kb marine medaka choriogenin $\mathrm{H}$ gene $(c h g H)$ promoter. The chgH-rfp transgene copies formed in a single site of the marine medaka genome were transmitted to multiple subsequent generations (now up to F5) following the Mendelian inheritance frequency without notable change of hybridizing band patterns in the genomic Southern blot analyses. No significant change was observed in the RFP phenotype with passing through generations as examined by epi-fluorescent microscopy or Western blot analysis. Of various estrogen-responsive transgenic lines generated in marine medaka, the transgenic strain used in this study was the stable line showing the strongest and the most sensitive response to both natural and synthetic versions of the estrogen compounds. Characteristics of this transgenic line [labeled TG\#038 in Cho et al. (2013)] in details could be referred to our previous reports (Cho et al. 2013; Nam et al. 2015).

\section{Fish rearing conditions}

Rearing and management of marine medaka were carried out according to the conditions described previously (Cho et al. 2010). Briefly, water temperature were kept at $25 \pm 1{ }^{\circ} \mathrm{C}$, and salinity was adjusted to $10 \mathrm{~g} \mathrm{~L}^{-1}$ adjusted using the synthetic sea salt (Kent Marine, GA, USA) throughout the experiments, including spawning of broodfish, incubation of developing embryos, larval nursery, grow-out trial, and estrogen exposure treatment. Photoperiod (daylight cycle) was 14-h light and 10-h dark. The yolk sac-absorbed larvae (about $40 \mathrm{~h}$ after hatching) were fed with $150-\mu \mathrm{m}$ artificial diets for flounder larvae (Woosung Feed Corp., Daejeon, Korea) and Artemia nauplii larvae. According to the growth of the fish, the size of the diets (same brand above) was increased gradually up to $500 \mu \mathrm{m}$. Daily water exchange rate was approximately $10-20 \%$ if not specifically defined.

\section{RFP phenotyping throughout life cycle under non- exposed conditions}

The $c h g H-r f p$ transgenic F4 offspring were produced in a hemizygous fashion by mating 60 F3 transgenic males (half-siblings) and 120 wild-type, non-transgenic females. In order to get embryos with synchronized development within a batch, each batch was prepared to contain 
about 1800-2000 fertilized eggs collected within the time window of $4 \mathrm{~h}$. Four batches were prepared. Each egg batch was transferred to an independent incubator, and the RFP signal was examined for at least $240 \mathrm{em}$ bryos with a 24-h interval by using the AZ100 fluorescence microscope (Nikon Corporation Instruments Company, Japan). In order to confirm the transgenic incidence in each batch, 48 embryos each randomly sampled at 2 days post fertilization (DPF; corresponding to the somite formation stage) and 10 DPF (pre-hatching stage) were subjected to PCR typing of the chgH-rfp transgene as described before (Cho et al. 2013).

We prepared two replicate hatchling batches in which each batch consisted of 1600-1800 hatched larvae collected within the time window of $6 \mathrm{~h}$. Each group of hatchlings was reared in net cages installed in 400-L water-recirculating rectangular tanks. In order to examine the naturally occurring RFP signal under nonexposed conditions, the fish were subjected to epifluorescence microscopy with a 1-week interval up to 10 weeks post hatching (WPH) (Song et al. 2009, 2010). Until the age of $4 \mathrm{WPH}$, live fish were directly examined with a fluorescent microscope because the fish during this early developmental period usually remained transparent enough to be detected for RFP signals under fluorescent microscopy. However, afterward, the abdominal cover (i.e., peritoneum) of the fish older than 4 weeks of age was surgically removed to better visualize the liver because the thickened abdominal cover might mask potentially the RFP signal. For each sampling point, at least 160 fish were assayed for RFP signals, and the total length of each individual was measured. The RFPnegative fish were subjected to PCR screening of transgene in order to exclude the non-transgenic individuals from the data analysis.

\section{Induction of RFP phenotype by estrogen exposures in transgenic male adults}

In order to examine if the adult transgenic males are able to respond to exogenously administered estrogen, transgenic males at $10 \mathrm{WPH}$ were experimentally exposed to two kinds of estrogens, estradiol-17 $\beta$ (E2) and $17 \alpha$-ethinylestradiol (EE2). Both compounds were purchased from Sigma-Aldrich (St. Louis, MO, USA). Because we aimed to examine primarily whether the estrogen-mediated RFP signal would be induced exclusively in the main target organ (i.e., liver) or ubiquitously also in other non-liver organs, we exposed transgenic males to high concentrations of estrogens $\left[1.0 \mu \mathrm{g} \mathrm{L} \mathrm{L}^{-1}\right.$ (for E2) and $0.5 \mu \mathrm{g} \mathrm{L}^{-1}$ (for EE2)]. These exposure conditions had been known to induce robustly endogenous chgH transcripts in male adults or RFP signals in transgenic larvae of this medaka species (Cho et al. 2013; Nam et al. 2015; Lee et al. 2012). We did not consider the evaluation of the lowest-observed-effect concentrations (LOECs) of E2 and EE2 here. For each exposure treatment, 24 transgenic males (PCR positive for their caudal fin DNA) and the same number of age-matched non-transgenic males were placed in one of two net cages $(30 \times 20 \times 15 \mathrm{~cm}=\mathrm{W} \times \mathrm{D} \times \mathrm{H})$ installed in the same tank $(90 \times 50 \times 25 \mathrm{~cm}=\mathrm{W} \times \mathrm{D} \times \mathrm{H})$. After a 24 -h acclimation period, they were exposed to estrogen at the desired concentration $\left(1.0 \mu \mathrm{g} \mathrm{L}^{-1} \mathrm{E} 2\right.$ or $0.5 \mu \mathrm{g} \mathrm{L}{ }^{-1} \mathrm{EE} 2$; nominal concentration) for 5 days. In order to prepare the non-exposed groups, 24 transgenic and 24 nontransgenic males were treated identically as described above except for the estrogen exposure. During exposure, fish were fed with a $500-\mu \mathrm{m}$ diet for flounder larvae (Woosung Feed Corp.) once per day. At 2.5 days post exposure, half of the water was exchanged and hormones were renewed at that time. At the end of the exposure, 10 kinds of tissues (brain, fin, gill, heart, intestine, kidney, liver, skeletal muscle, spleen, and testis) were surgically sampled from each individual in order to examine the induction of RFP signals with the fluorescent microscopy. The arbitrary values of RFP signals (statistical mean of intensity values of the pixels) were measured using the NIS-Elements BR Image software (ver. 3.1) equipped in the AZ 100 fluorescence microscope system (Nikon).

\section{Results and discussion}

PCR typing indicated that the transgene was successfully transmitted to the F4 generation, as evidenced by the incidence of transgenic fish close to $50 \%$ (52\% in average for embryonic samples and 51\% for post-hatch samples), confirming clearly our previous result on the stable germline transmission in a hemizygous status of this selected transgenic marine medaka strain (Cho et al. 2013). Overall hatching success was $89.5 \pm 4.7 \%$ on average from the four egg batches. During the embryonic development until hatching, none of the transgenic embryos showed a naturally occurring RFP signal (data not shown). The absence of the RFP signal during embryonic development was not congruent with the endogenous mRNA expression of the $c h g H$ gene. Based on the RT-PCR, endogenous $c h g H$ transcripts have been reported to be observable in developing embryos notably from the visceral blood vessel formation stage (Lee et al. 2012), which corresponds to the stage of visible liver formation and active blood circulation in the Oryzias species (Song et al. 2009; Ueno et al. 2004; Chen et al. 2008). It suggests that the transgene regulation might not resemble exactly that of the endogenous $c h g H$ gene during embryonic development. Alternatively, the amount of RFP accumulated might not be enough to be detected by the fluorescent microscopy. Differential expression patterns of the transgene under the control of the same 
endogenous gene promoter as compared to the expression of the endogenous gene have been reported in several transgenic fish strains (Cho et al. 2011; Lee et al. 2013).

After hatch-out, the transgene was tightly kept to be silent in the early developmental stages under nonexposed conditions, as indicated by the absence of the RFP signal (Table 1). During this period, phenotypic sexing by examining the external morphology of fish was almost impossible. Previous studies have reported that the Oryzias species should possess previtellogenic (or early vitellogenic) oocytes in ovaries at the period from 4 to $5 \mathrm{WPH}$ while spermatids become observable in testes at 4 WPH (Song 2010; Kinoshita et al. 2009). At $6 \mathrm{WPH}$, an apparent, but not significantly matured, ovarian or testicular shape could be identified with the unaided eye in a few individuals and gonadal sexing of each fish was possible based on the microscopic examination of squashed gonad samples. Medaka fishes at this age have been known to possess vitellogenic oocytes with developed yolk vesicles in females (Kinoshita et al. 2009). However, in the present study, none of transgenic females showed naturally occurring RFP signals, suggesting that the regulation of transgenic chgH would not be robust yet in sexually differentiated, but immature, transgenic fish, which is generally in accordance with the anticipated role of the choriogenin as the main constitute of egg envelop (Hong et al. 2009). However, these young immature individuals have been proven to respond readily to exogenously administered estrogens as reported previously (Cho et al. 2013; Nam et al. 2015).

At $7 \mathrm{WPH}$, a number of individuals showed discernable ovaries and testes that could be easily identified with naked eyes. Histologically, marine medaka has been reported to possess either ovaries containing developing oocytes with apparent yolk granulations or testes containing considerable number of spermatids with some spermatogonia (Song 2010; Kinoshita et al. 2009). Notably, a small proportion of transgenic females $(7.7 \%$ for trial I and 7.5\% for trial II) begun to show RFP signals in their livers. On the other hand, none of transgenic males displayed RFP signals at this age (Table 1). From 8 WPH, sexing was highly feasible based on only direct examination of the gonad with the naked eye. The sex

Table 1 Incidence of naturally occurring RFP signals in the liver of transgenic marine medaka with the function of age

\begin{tabular}{|c|c|c|c|c|c|}
\hline \multirow{2}{*}{$\begin{array}{l}\text { Age (weeks } \\
\text { post hatching) }\end{array}$} & \multirow{2}{*}{$\begin{array}{l}\text { No. of TG } \\
\text { fish examined }\end{array}$} & \multirow{2}{*}{$\begin{array}{l}\text { Average total } \\
\text { length }(\mathrm{mm})\end{array}$} & \multicolumn{3}{|c|}{ \% RFP-positive fish (no. of RFP-positive fish/no. of TG fish) } \\
\hline & & & Female & Male & Not sexed \\
\hline \multicolumn{6}{|l|}{ Trial I } \\
\hline 1 & 75 & $7.9 \pm 0.2$ & - & - & $0.0(0 / 75)$ \\
\hline 2 & 74 & $10.2 \pm 0.4$ & - & - & $0.0(0 / 74)$ \\
\hline 3 & 79 & $10.8 \pm 0.4$ & - & - & $0.0(0 / 79)$ \\
\hline 4 & 81 & $12.9 \pm 0.6$ & - & - & $0.0(0 / 81)$ \\
\hline 5 & 72 & $15.2 \pm 1.0$ & - & - & $0.0(0 / 72)$ \\
\hline 6 & 79 & $17.5 \pm 1.2$ & $0.0(0 / 39)$ & $0.0(0 / 40)$ & - \\
\hline 7 & 82 & $19.1 \pm 1.5$ & $7.7(3 / 39)$ & $0.0(0 / 43)$ & - \\
\hline 8 & 85 & $21.8 \pm 1.4$ & $47.6(20 / 42)$ & $0.0(0 / 43)$ & - \\
\hline 9 & 76 & $23.1 \pm 2.1$ & $75.0(27 / 36)$ & $0.0(0 / 40)$ & - \\
\hline 10 & 87 & $25.2 \pm 2.2$ & $95.6(43 / 45)$ & $0.0(0 / 42)$ & - \\
\hline \multicolumn{6}{|l|}{ Trial ॥ } \\
\hline 1 & 85 & $7.5 \pm 0.2$ & - & - & $0.0(0 / 85)$ \\
\hline 2 & 83 & $9.9 \pm 0.3$ & - & - & $0.0(0 / 83)$ \\
\hline 3 & 88 & $10.2 \pm 0.5$ & - & - & $0.0(0 / 88)$ \\
\hline 4 & 85 & $12.5 \pm 0.5$ & - & - & $0.0(0 / 85)$ \\
\hline 5 & 87 & $15.0 \pm 1.0$ & - & - & $0.0(0 / 87)$ \\
\hline 6 & 85 & $17.1 \pm 1.2$ & $0.0(0 / 40)$ & $0.0(0 / 45)$ & - \\
\hline 7 & 79 & $19.3 \pm 1.3$ & $7.5(3 / 40)$ & $0.0(0 / 39)$ & - \\
\hline 8 & 81 & $20.9 \pm 1.5$ & $55.0(22 / 40)$ & $0.0(0 / 41)$ & - \\
\hline 9 & 86 & $22.8 \pm 2.0$ & $70.7(29 / 41)$ & $0.0(0 / 45)$ & - \\
\hline 10 & 85 & $24.8 \pm 2.1$ & $100.0(44 / 44)$ & $0.0(0 / 41)$ & - \\
\hline
\end{tabular}

Number of transgenic fish was estimated based on the PCR typing of RFP-negative fish. Gonadic sex of fish at 6 WPH and 7 WPH was judged based on the microscopic examination of squashed gonad specimens, while sexing of the fish at $8 \mathrm{WPH}$ to $10 \mathrm{WPH}$ was done by direct examination of gonads with the unaided eye 


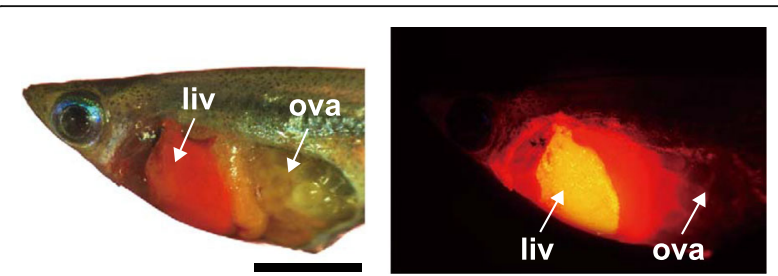

Fig. 1 A 10-week-old chgH-rfp transgenic marine medaka Oryzias dancena female at full maturity. RFP signals were expressed in the liver with the progressive maturation of oocytes in the ovary (ova). Under the daylight condition (left), the reddish liver was apparently seen and intensified red fluorescence in the liver of the same female was visualized under the dark field of microscopy (right). The bar is $5 \mathrm{~mm}$

ratio was close to $1: 1 \quad(P>0.05$ with chi-square test), which was in agreement with previous observation (Song et al. 2010). In this maturation stage, a number of individuals displayed well-developed ovaries containing yolkladen eggs (often ovulated eggs from $9 \mathrm{WPH}$ ) or milky white testes filled with spermatids and spermatozoa. Usually around this age, the sex-related dimorphism is also easily observable for the morphology of the anal fin (Song et al. 2009). Occurrence patterns of RFP signals were apparently different depending upon sexes. In females, the percent RFP-positive individuals were sharply increased with ages (from 47.6-55.0\% at $8 \mathrm{WPH}$ to 95.6-100.0\% at $10 \mathrm{WPH}$ ), suggesting that endogenous estrogen signaling should activate the chgH promoter with the progress of ovarian maturation and ovulation. This finding is in agreement with the previous result that reported that the first spawning of the female usually occurs at 9-10 WPH (Song et al. 2009; Song et al. 2010). At $10 \mathrm{WPH}$, fully matured females often resulted in the reddish livers even under daylight condition due to the largely accumulated amounts of RFPs. The glowing red fluorescent signal in these livers could be visualized under fluorescence microscopic illumination (Fig. 1). However, in contrast, none of the chgH-rfp transgenic males exhibited the RFP signal even after they developed fully matured testes containing lots of spermatozoa. Previously, marine medaka males have been reported to express small amounts of chgH mRNAs exclusively in the liver under non-exposed conditions (Lee et al. 2012); however, the present finding has indicated such transcription may not attribute significantly to transgenic phenotype. Collectively, unlike the female showing natural occurrence of RFP signals with the progress of maturation, chgH-rfp transgenic males could keep the "off" status of the transgenic signal (i.e., RFP-negative) throughout their entire life cycle under non-exposed conditions.

Based on the examination above, transgenic male adults were subjected to experimental estrogen exposures (E2 and EE2) in order to scrutinize the pattern of estrogen-responsive induction of transgenic RFP signals. As expected, non-transgenic males consistently exhibited the RFP-negative phenotype irrespective of estrogen treatments. Again, transgenic males that were not exposed to estrogen also did not show any naturally occurring RFP signals. Background signals (mean of intensity values of the pixels) measured with these RFP-negative fishes were less than the arbitrary value of 5.0. However, upon E2 or EE2 exposure, chgH-rfp transgenic males expressed and accumulated RFPs in their livers (Fig. 2). All the exposed transgenic males displayed strong RFP induction without exception, in which mean intensity values of RFP signals were $295.8 \pm 75.6$ and $341.9 \pm 81.5$ for E2- and EE2-exposed groups, respectively. Although there was no statistical difference in the arbitrary values of RFP signals between E2- and EE2-exposed transgenic males $(P>0.05$; Student's $t$ test), the potency of EE2 to activate the chgH-rfp transgene expression might be greater than that of E2 in transgenic marine medaka males, since the treatment dose of EE2 used in this study was only the half of E2. EE2, an orally bioactive estrogen, has been known to possess much higher estrogenic potency than E2 (Kurauchi et al. 2005; Kurauchi et al. 2008); the present finding is similar to our previous

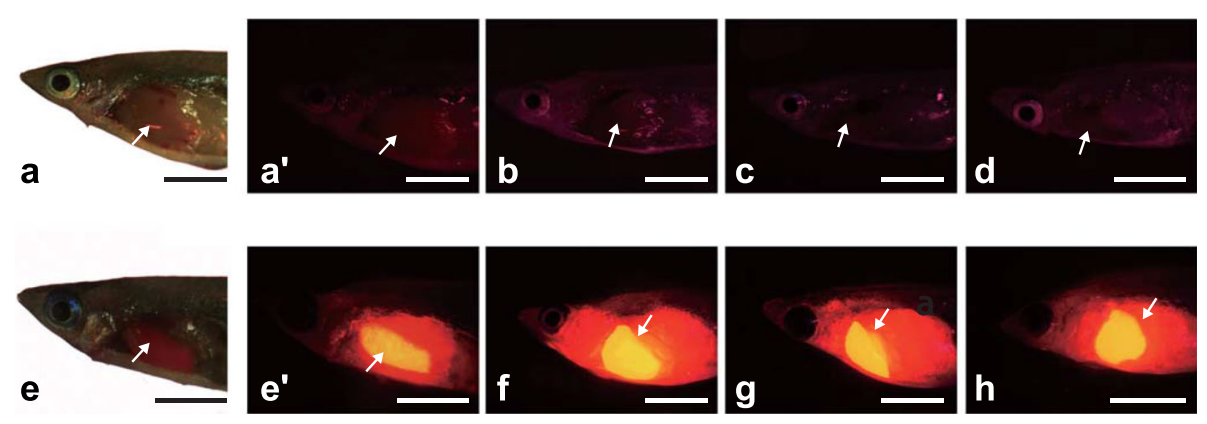

Fig. 2 Representative images to show the transgenic induction of RFP signals in response to estrogen exposures in the liver of chgH-rfp transgenic marine medaka Oryzias dancena males. a-c Non-exposed transgenic males. d E2-exposed non-transgenic male. e, $\mathbf{f}$ E2-exposed transgenic males. $\mathbf{g}, \mathbf{h}$ EE2-exposed transgenic males. Photographs $\mathbf{a}^{\prime}$ and $\mathbf{e}^{\prime}$ are dark-field images of $\mathbf{a}$ and $\mathbf{e}$, respectively. Arrows indicate livers. The bars are $5 \mathrm{~mm}$ 
observation using chgH-rfp transgenic marine medaka larvae (Cho et al. 2013).

In RFP-positive transgenic males from estrogentreated groups, the induction of the transgenic RFP signal was exclusively observed in the liver while the other nine kinds of tissues examined showed no clear sign of RFP induction under the present microscopic conditions (photograph not shown). This finding is incongruent with previous finding in the sense that experimental exposure of E2 could induce de novo synthesis of chgH transcripts in non-liver tissues such as the kidney, spleen, and heart (Lee et al. 2012). Because we examined the expression of the transgene only at the protein level (RFP signal), further study is needed to examine whether the RFP-negative tissues in the present study express $r f p$ transcripts or not. Differential regulation and/or deregulation of the endogenous gene of which the promoter is used as the transgenic regulator has been reported in transgenic animals particularly having multiple copy transgene integrants where transgene and endogenous gene promoters could be potentially competitive for the binding of essential transcription factors (Cho et al. 2011; Kumar et al. 2004).

Taken together, marine medaka chgH-rfp transgenic males could induce transgenic expression in response to estrogen exposures in a highly tissue-specific fashion without the leaking of the RFP signal under nonexposed conditions. However, further extensive investigations are needed in order to evaluate the functionality and usefulness of these transgenic marine medaka males for detecting waterborne estrogenicity in detail especially regarding the LOECs of various potential estrogenic pollutants in a more environmentally realistic fashion.

\section{Conclusion}

Expression patterns of the chgH-rfp transgene at different developmental stages and reproductive statue were examined in female and male marine medaka $O$. dancena. Based on RFP phenotyping during the life cycle, female transgenics begun to show naturally occurring RFP signals from the age of $7 \mathrm{WPH}$ under non-exposed conditions, and afterwards, these RFP signals became intensified with the progress of ovarian maturation. On the other hand, male transgenics did not show any leaking of RFP signals during their entire life cycle, unless exposed to estrogens. Induction of RFP signals in male transgenics were sensitively achieved in response to E2 and EE2 exposures, and the induced pattern was highly liver specific. Data from this study could be a good basis to designate various future studies regarding ecotoxicogenomic applications of estrogen-responsive transgenic medaka.

\section{Abbreviations}

ChgH: Choriogenin H gene; ChgH-rfp: Red fluorescent protein transgene driven by choriogenin H promoter; E2: Estradiol-17ß; EE2: 17a-

Ethinylestradiol; RFP: Red fluorescent protein

\section{Acknowledgements}

The authors thank Mr. Byoung Soo Kim for his technical assistance on the management and sampling of experimental fish specimens.

\section{Funding}

This study was supported by the grant from Pukyong National University (year 2015).

\section{Availability of data and materials \\ Not applicable}

\section{Authors' contributions}

YSC carried out the transgene analysis and expression assay. YKN designed the study and data evaluation and drafted the manuscript. Both authors read and approved the final manuscript.

\section{Competing interests}

The authors declare that they have no competing interests.

\section{Consent for publication}

Not applicable

Ethics approval and consent to participate

Not applicable

Received: 5 August 2016 Accepted: 16 November 2016 Published online: 25 November 2016

\section{References}

Bogers R, Mutsaerds E, Druke J, De Roode DF, Murk AJ, Van Der Burg B, Legler J. Estrogenic endpoints in fish early life-stage tests: luciferase and vitellogenin induction in estrogen-responsive transgenic zebrafish. Environ Toxicol Chem. 2006;25:241-7.

Chen X, Li WWT, Yu RMK, Cheng SH. Choriogenin mRNA as a sensitive molecular biomarker for estrogenic chemicals in developing brackish medaka (Oryzias melastigma). Ecotoxicol Environ Saf. 2008;71:200-8.

Chen H, Hu J, Yang J, Wang Y, Xu H, Jiang Q, Gong Y, Gu Y, Song H. Generation of a fluorescent transgenic zebrafish for detection of environmental estrogens. Aquat Toxicol. 2010;96:53-61.

Cho YS, Lee SY, Kim DS, Nam YK. Spawning performance, embryonic development and early viability under different salinity conditions in a euryhaline medaka species, Oryzias dancena. Korean J Ichthyol. 2010;22:25-33.

Cho YS, Lee SY, Kim YK, Kim DS, Nam YK. Functional ability of cytoskeletal $\beta$-actin regulator to drive constitutive and ubiquitous expression of a fluorescent reporter throughout the life cycle of transgenic marine medaka Oryzias dancena. Transgenic Res. 2011;20:1333-55.

Cho YS, Kim DS, Nam YK. Characterization of estrogen-responsive transgenic marine medaka Oryzias dancena germlines harboring red fluorescent protein gene under the control by endogenous choriogenin $\mathrm{H}$ promoter. Transgenic Res. 2013;22:501-17.

Hong L, Fujita T, Wada T, Amano H, Hiramatsu N, Zhang X, Todo T, Hara A. Choriogenin and vitellogenin in red lip mullet (Chelon haematocheilus): purification, characterization, and evaluation as potential biomarkers for detecting estrogenic activity. Comp Biochem Physiol C Toxicol Pharmacol. 2009;149:9-17.

Kinoshita M, Murata K, Naruse K, Tanaka M. Reproduction of medaka. In: Kinoshita M, Murata K, Naruse K, Tanaka M, editors. Medaka: biology, management, and experimental protocols. lowa: Wiley-Blackwell; 2009. p. 67-99.

Kumar A, Crawford K, Flick R, Klevitsky R, Lorenz JN, Bove KE, Robbins J, Lessard $J \mathrm{~L}$. Transgenic overexpression of cardiac actin in the mouse heart suggests coregulation of cardiac, skeletal and vascular actin expression. Transgenic Res. 2004;13:531-40.

Kurauchi K, Nakaguchi Y, Tsutsumi M, Hori H, Kurihara R, Hashimoto S, Ohnuma R, Yamamoto Y, Matsuoka S, Kawai S, Hirata T, Kinoshita M. In vivo visual reporter system for detection of estrogen-like substances by transgenic medaka. Environ Sci Technol. 2005;39:2762-68. 
Kurauchi K, Hirata T, Kinoshita M. Characteristics of ChgH-GFP transgenic medaka lines, an in vivo estrogenic compound detection system. Mar Pollut Bull. 2008:57:441-4.

Lee SY, Kim DS, Nam YK. Gene structure and estrogen responsive mRNA expression of a novel choriogenin $\mathrm{H}$ isoform from a marine medaka (Oryzias dancena). Fish Aquat Sci. 2012;15:221-31.

Lee SY, Kim DS, Nam YK. Molecular characterization of fast skeletal musclespecific myosin light chain 2 gene $(\mathrm{m} / \mathrm{c} 2 \mathrm{f})$ in marine medaka Oryzias dancena. Genes Genom. 2013;35:289-303.

Nam YK, Cho YS, Kim DS. Expression patterns of the chgH:rfp transgene in response to 17a-ethinylestradiol (EE2) exposure in marine medaka Oryzias dancena. Fish Aquat Sci. 2015;18:65-71.

Salam MA, Sawada T, Ohya T, Ninomiya K, Hayashi S. Detection of environmental estrogenicity using transgenic medaka hatchlings (Oryzias latipes) expressing the GFP tagged choriogenin L gene. J Environ Sci Health, Part A. 2008;43:272-7.

Scholz S, Kurauchi K, Kinoshita M, Oshima Y, Ozato K, Schirmer K, Wakamatsu Y. Analysis of estrogenic effects by quantification of green fluorescent protein in juvenile fish of a transgenic medaka. Environ Toxicol Chem. 2005;24:2553-61.

Song HY. Cytogenetic and reproductive characteristics of interspecific hybrids between marine medaka Oryzias dancena and Javanese medaka O. javanicus. Ph.D. Dissertation Pukyong Natl Univ. Busan. 2010. p. 133.

Song HY, Nam YK, Bang IC, Kim DS. Embryogenesis and early ontogenesis of a marine medaka, Oryzias dancena. Korean J Ichthyol. 2009;21:227-38.

Song HY, Nam YK, Bang IC, Kim DS. Hybridization between marine medaka Oryzias dancena and Javanese medaka Oryzias javanicus. Kor J Fish Aquat Sci. 2010;43:462-73

Stegeman JJ, Goldstone JV, Hahn ME. Perspectives on zebrafish as a model in environmental toxicology. Fish Physiol. 2010;29:367-439.

Ueno T, Yasumasu S, Hayashi S, luchi I. Identification of choriogenin cis-regulatory elements and production of estrogen-inducible, liver-specific transgenic medaka. Mech Dev. 2004;121:803-15.

Zeng Z, Shan T, Tong Y, Lam SH, Gong Z. Development of estrogen-responsive transgenic medaka for environmental monitoring of endocrine disrupters. Environ Sci Technol. 2005;39:9001-8.

\section{Submit your next manuscript to BioMed Central and we will help you at every step:}

- We accept pre-submission inquiries

- Our selector tool helps you to find the most relevant journal

- We provide round the clock customer support

- Convenient online submission

- Thorough peer review

- Inclusion in PubMed and all major indexing services

- Maximum visibility for your research

Submit your manuscript at www.biomedcentral.com/submit

Biomed Central 